\title{
Discussion of the relationship between failure and fire of valve regulated lead acid battery
}

\author{
Zhenyu $\mathrm{Li}^{1 *}$, Zhongjie Wang ${ }^{1}$, Liang Wang ${ }^{2}$ \\ ${ }^{1}$ State Grid Shanxi Electric Power Company, Taiyuan, Shanxi, 030021, China \\ ${ }^{2}$ State Grid Shanxi Electric Power Research Institute of SEPC, Taiyuan, Shanxi, 030001, China
}

\begin{abstract}
Failure modes of the valve regulated lead acid battery will not only greatly reduce the service life, but also may start a fire. This paper reviews the relationship between battery fire and failure modes. Four failure modes influenced on the valve regulated lead acid battery were emphatically analyzed: "Sulfation of negative electrode plate", "corrosion of the positive electrode plate", "loss of water" and "acid leak". The direct reasons for battery fire are thermal runaway, short circuit and hydrogen explosion, which were inducing by battery failure. A fire prevention scheme was proposed on the basis of above thesis.
\end{abstract}

\section{Introduction}

Valve-regulated lead-acid (VRLA) battery has been widely used in communication power supply system and UPS power system, owing to the advantage of low price, convenient maintenance, high reliability and so on. Furthermore, a series of VRLA batteries can be used as an emergency power, which plays an extremely important role in the safe and stable operation of the power system. With the development of smart grids, unattended substation has been the popular trend in recent years, but the battery fire accidents caused by failure modes still occurred sometimes. This means that the accurate analysis of the correlation between battery failure modes and fire is an urgent priority event to ensure the safe operations of substations. Some researchers have studied the relationship between the early failure modes (e.g. irreversible sulfation, corrosion of the positive electrode plate, loss of water and acid leak) and batter fire [1-3]. Their results show that the risk of thermal runaway and short circuit of faulty batteries is significantly higher than that of new batteries.

This paper combines with the three factor of combustion to study the relationship among the common failure modes, thermal runaway and short circuit of VRLA battery, and proposes the fire prevention programs accordingly.

\section{Common failure modes of VRLA battery}

With the increasing charge and discharge frequency of VRLA battery, it is usually accompanied by early failure phenomena such as the drop of cell voltage and capacity. Such occurred situations indicate that the battery has suffered some failures such as sulfation of negative electrode plate, corrosion of the positive electrode plate, loss of water and acid leak, which will be explained in detail in the following four subsections.

\subsection{Sulfation of negative electrode plate}

Battery sulfating refers to the fact that the $\mathrm{PbSO}_{4}$ crystals are formed on the negative electrode plate, caused by insufficiently charged, over discharge for a long time and naked negative electrode plate due to the lack of electrolyte. The $\mathrm{PbSO}_{4}$ crystals with poor conductivity will block the micropores of the electrode plate, hinder the penetration of the electrolyte and increase the internal resistance. After that, the Joule heat increases with increasing floating charging current, leading to battery thermal runaway easily. The research by Bouabidi et al. [4] showed that irreversible sulfation of the negative electrode plate not only decreased the capacity of battery directly, but also produced the white-gray precipitates on the battery tank or electrode, finally bending and deforming the negative electrode plate. Valenciano et al. [5] studied the distribution of sulfates in the negative electrode plate of lead-acid batteries before and after failure. They found that the corrosion of the upper part of the negative electrode plate was more serious than the lower part, resulting in the bending and of electrode plate.

\subsection{Corrosion of the positive electrode plate}

The positive electrode plate will be corroded by battery overchange, which performs that $\mathrm{Pb}$ is oxidized to $\mathrm{PbO}_{2}$ [6]. The equations are presented as follow:

$$
\mathrm{PbO}+\mathrm{H}_{2} \mathrm{O} \rightarrow \mathrm{PbO}_{2}+2 \mathrm{H}^{+}+2 e
$$

*Corresponding author's e-mail: 1zy710312@sohu.com 


$$
\begin{aligned}
& \mathrm{PbO}+\mathrm{H}_{2} \mathrm{O} \rightarrow \mathrm{PbO}_{2}+2 \mathrm{H}^{+}+2 e \\
& \mathrm{~Pb}+2 \mathrm{H}_{2} \mathrm{O} \rightarrow \mathrm{PbO}_{2}+2 \mathrm{H}^{+}+2 e
\end{aligned}
$$

The battery resistance increases by the corrosion of positive electrode plate, possibly due to the reduced contact area between the effective substance and the electrode plate. The research by Chen et al. [7] showed that the corrosion of positive electrode plate and the connection bar between negative and positive electrode reduce the metal channel of the battery, leading to an increase in the battery resistance. Moreover, Ball et al. [8] studied the varied content of $\alpha-\mathrm{PbO}_{2}$ and $\beta-\mathrm{PbO}_{2}$ in both normal and failed battery, and they found that the shedding of active materials is directly related to the conversion between $\alpha-\mathrm{PbO}_{2}$ and $\beta-\mathrm{PbO}_{2}$. In detail, the $\alpha$ $\mathrm{PbO}_{2}$ in the failed battery is much lower than that of the normal battery, which is believed that loss of $\alpha-\mathrm{PbO}_{2}$ is the main reason for the softening and failure of battery. Overall, the corrosion of the positive electrode plate increases the internal resistance of the battery, while decreasing the battery capacity and cycle life.

\section{3 "Water loss" of battery}

The negative electrode plate material used in VRLA battery is $\mathrm{Pb}-\mathrm{Sb}$ alloy normally. At the end of charring, the safety valve opened, along with a small amount of water and acid are taken away with the hydrogen evolution. Chen et al. [9] summarized the reasons for the battery dehydration, and they believed that the water loss was mainly owing to poor gas recombination efficiency, high floating charge pressure, poor battery sealing and overcharge, etc. After analyzing the status of VRLA battery as a result of long-term operation and maintenance, Yang et al. [10] concluded that the ambient temperature, float voltage, overcharge and corrosion of the positive electrode plate are related with battery dehydration. In addition, the battery dehydration will increase the resistance of conductive channel, Joule heating, and decreasing the thermal capacity of battery. Hu et al. [11] studied the relationship between dehydration and thermal runaway of battery, and found that the electrolyte saturation decreases as the degree of water loss (especially for the electrolyte saturation is less than $85 \%$ ), and thus increasing the risk of thermal runaway significantly. The reason is that the oxygen diffusion resistance increase with the electrolyte saturation, due to the fewer gas channels left in this condition. In contrast, the oxygen easily moves to the negative electrode plate for redox reaction, releasing extra thermal energy, and thereby supporting the battery thermal runaway. Wang et al. [12] found that about $80 \%$ of the battery thermal capacity is decided by the electrolyte, which is constitute of water. According to the definition of heat capacity $\mathrm{C}(\mathrm{C}=\sigma \mathrm{Q} / \mathrm{dT})$, it can be concluded that the battery with low heat capacity is easier to heat up, and therefore increasing the risk of thermal runaway when battery suffered water loss.

\subsection{Acid leak of battery}

The leakage of VRLA battery is mainly related to battery thermal runaway, which is induced by excessive electrolyte injection, inadequate sealing, unqualified sealing materials and aging of sealing materials, etc. The manufacturing defects and operation mistakes including acid leakage from the safety valve, leakage from the pole column, failure of safety valve, lower valve opening pressure, and too high charging safety. In addition, the battery shell will be softened by thermal runaway, destroying the battery structure and causing a large amount of acid leak out.

\section{Effects of failure modes on thermal runaway and short circuit of battery}

In the absence of an external heat source, the increasing temperature of battery mainly depends on battery thermal runaway and short circuit. During the charging process of the battery, the oxygen generated by the positive electrode plate moves to negative electrode plate as shown in Figure 1.

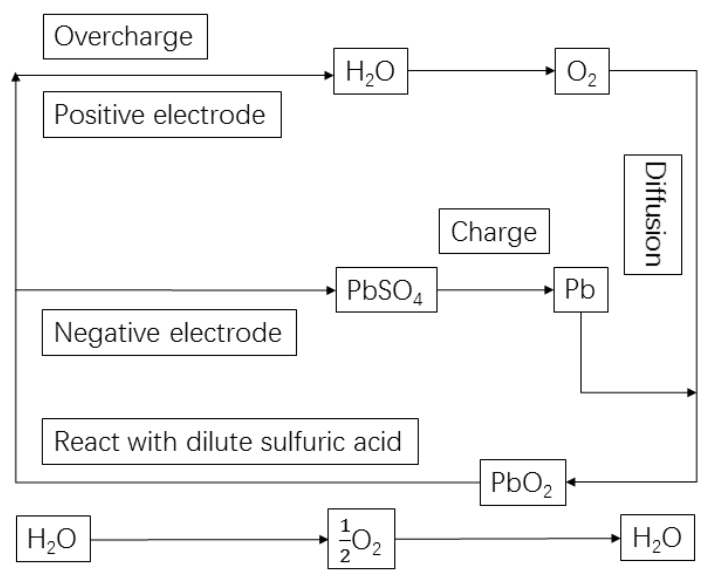

Figure 1. The closed oxygen cycle [13]

It can be seen in Eq. 4 and Eq. 5 that the redox reaction releases numerous heat, heating up the battery. The increasing temperature also accelerates the oxygen cycle ratio and the charging current. The vicious circle creates the greater internal heat generation rate than heat dissipation rate, causing the battery temperature continuous rise, finally leading to thermal runaway [13].

$$
\begin{aligned}
\mathrm{Pb}+\mathrm{O}_{2} \rightarrow \mathrm{PbO} \Delta \mathrm{H} \\
=-219.2 \mathrm{~kJ} / \mathrm{mol} \\
\mathrm{PbO}+\mathrm{H}_{2} \mathrm{SO}_{4} \\
+\mathrm{PbSO}_{4} \\
+\mathrm{H}_{2} \mathrm{O} \Delta \mathrm{H} \\
=-172.8 \mathrm{~kJ} / \mathrm{mol}
\end{aligned}
$$

Berndt et al. [14] conducted a step-by-step measurement of generated heat during charge and discharge of the VRLA battery, and they found that the total heat generated by the battery was $31.2 \mathrm{~W} \cdot \mathrm{h}$, which 
contained the reversible thermal effect of batter main reaction $(5.7 \mathrm{~W} \cdot \mathrm{h})$, the Joule heat $(2.3 \mathrm{~W} \cdot \mathrm{h})$ and the heat of closed oxygen circulation $(23.2 \mathrm{~W} \cdot \mathrm{h})$. That means about $3 / 4$ total heat is generated by the closed oxygen circulation. Tao et al. [15] have used twice the normal charging current to a new VRLA battery. They found that the temperature around battery all increased with the charging time. The temperature of battery when charging for $10 \mathrm{~h}$ are $70^{\circ} \mathrm{C}, 65^{\circ} \mathrm{C}, 78^{\circ} \mathrm{C}, 78^{\circ} \mathrm{C}$ for the front, upper, left and right, respectively, closes or exceeding the heatresistant temperature $\left(74^{\circ} \mathrm{C}-85^{\circ} \mathrm{C}\right)$ of the battery cases material (ABS resin). The battery thermal runaway usually related to the occurrence of short circuit. The spark caused by short circuit is often regarded as the ignition resource of hydrogen, resulting in battery explosion.

The relationships among batter failure, thermal runaway and short circuit were presented in Figure 2. The summarized factors for battery failure and runaway including: (1) The increasing battery resistance caused by sulfation of negative electrode plate and the corrosion of positive electrode plate, producing the Joule heat according to the formula $\left(\mathrm{Q}=\mathrm{I}^{2} \mathrm{Rt}\right)$; (2) The dehydration decreases the heat capacity of battery, while increasing its heating rate; (3) The corrosion of positive electrode plate will consume water and increase the oxygen channel in separator, releasing extra heat and disrupting the heat balance, which in turn leading to battery thermal runaway.

Another three factors were summarized for battery failure and short circuit: (1) The bending negative electrode plate and the inflated positive electrode plate were caused by irreversible sulfation and corrosion, respectively, damaging the separator and leading to the contact between positive electrode and negative electrode and forming the short circuit. (2) The active material falls from corrosive positive electrode plate and precipitates to a "conductive layer", then forming a short circuit; (3) The battery sulfation decrease the electrolyte saturation with the charging progresses. According to the common ion effect, part of the lead ion dendrites and converts into led metal to form lead dendrites [16], finally connecting the positive and negative electrode as well as producing a short circuit.

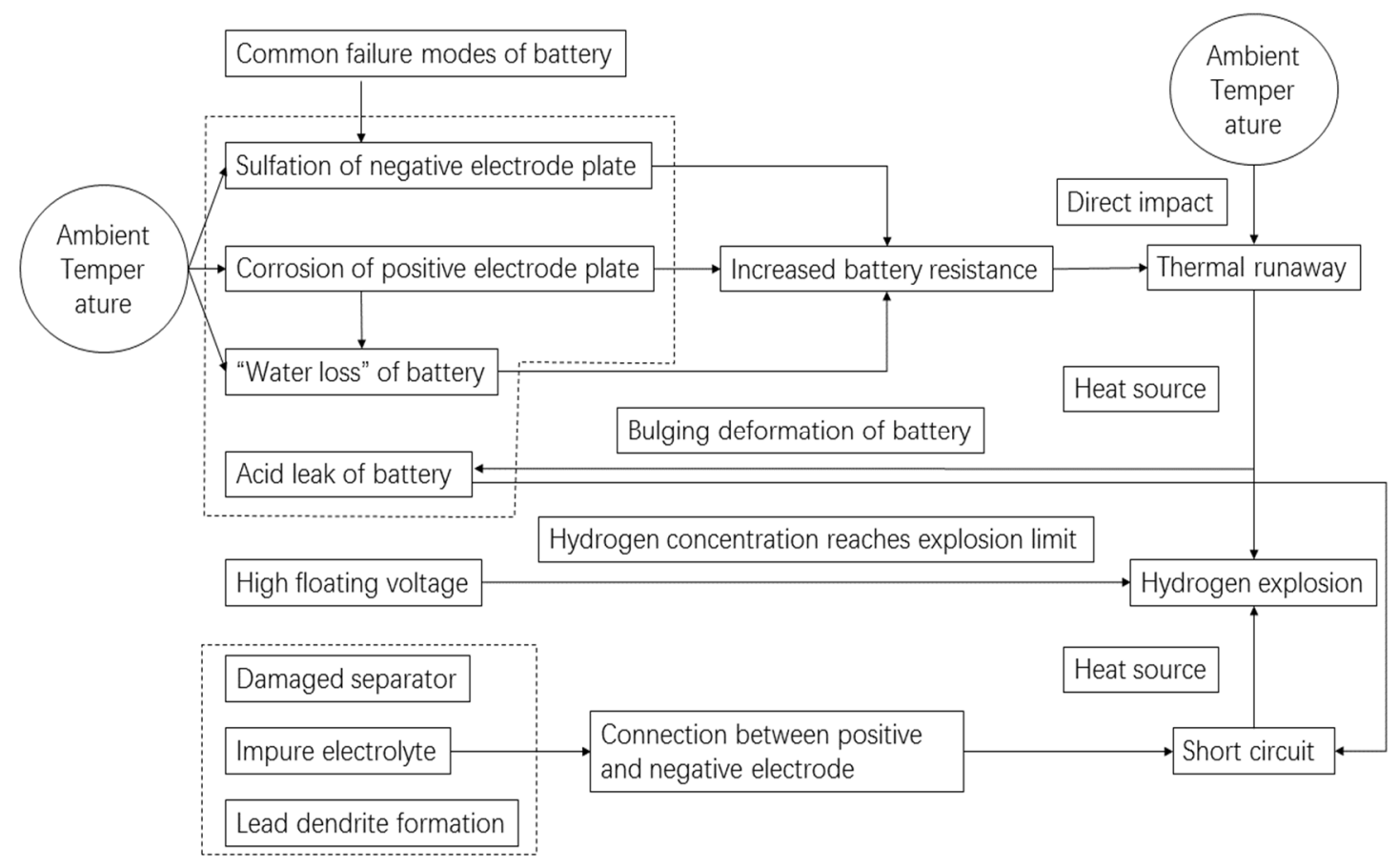

Figure 2. The association of VRLA battery failure with thermal runaway and short circuit

\section{Effects of failure modes on hydrogen and oxygen evolution of battery}

Besides the battery short circuit and thermal runaway, another significant reason for battery fire is the explosion caused by hydrogen evolution. Therefore, it is of great importance to analyze the properties of the gas component generated during both charging and discharging process. Liu et al. [17] analyzed the gas composition of lead-acid battery without temperature compensation, and they found only hydrogen and oxygen during the floating charging conditions. Similarly, Yang et al. [18] studied the reason for the hydrogen and oxygen evolution of lead-acid battery. The oxygen was believed to come from the closed oxygen cycle that can't be fully absorbed by the negative electrode plate. Moreover, the hydrogen is generally produced by the self-discharge reaction between metal impurities in negative electrode plate and sulfuric acid solution. The another explanation for that phenomenon is the negative electrode plate starts to evolve hydrogen at the end of charging. As can be seen in Figure 1, the research by Lam et al. [19] gave the limited content of 17 metal elements such as nickel, antimony, and cobalt, etc before hydrogen evolution in the negative electrode plate. 
Since the $\mathrm{Pb}-\mathrm{Sb}$ alloys, $\mathrm{Pb}-\mathrm{Ca}$ alloys, $\mathrm{Pb}-\mathrm{Sb}$-Sn-based alloys, and $\mathrm{Pb}-\mathrm{Ca}-\mathrm{Sn}-\mathrm{Al}$ allolys are the commonly used materials of negative electrode, it should be particularly pay attention to the effect of these metal element additives on the self-discharge reaction on the negative electrode plate. It was found by Long et al. [20] that the presence of bismuth could prevent the hydrogen evolution in negative electrode plate, while improving the recombination efficiency of oxygen in positive electrode plate. This results provide a good solution to prevent battery failure and hydrogen explosion fire.

Table 1. The maximum allowable impurity content of the negative electrode plate in lead-acid battery $(\mathrm{mg} / \mathrm{kg})^{[19]}$

\begin{tabular}{cccccccccccccccccc} 
Components & $\mathrm{Ni}$ & $\mathrm{Sb}$ & $\mathrm{Co}$ & $\mathrm{Cr}$ & $\mathrm{Fe}$ & $\mathrm{Mn}$ & $\mathrm{Cu}$ & $\mathrm{Ag}$ & $\mathrm{Se}$ & $\mathrm{Te}$ & $\mathrm{Ti}$ & $\mathrm{As}$ & $\mathrm{Sn}$ & $\mathrm{Bi}$ & $\mathrm{Ge}$ & $\mathrm{Zn}$ & $\mathrm{Cd}$ \\
Concentrations & 4 & 5 & 4 & 7 & 10 & 5 & 34 & 66 & 1 & 0.5 & 25 & 5 & 40 & 500 & 10 & 500 & 500 \\
\hline
\end{tabular}

To the author's knowledge, the hydrogen and oxygen are produced by the electrolysis of water, especially for overcharge battery. With the gas product accumulation, the internal pressure surpasses the critical value, opening the safety valve and taking part of the water and acid liquid to the environment. As a result, the hydrogen concentration around the battery may reach the explosion limit range of $4 \%$ to $75.6 \%$, possibly causing hydrogen explosion. However, the results by Wang et al. [21] showed that the maximum hydrogen concentration of 7500Ah, 79200Ah and 162000Ah capacity for VRLA battery was $4144 \mathrm{ppm}$, which was much lower than the explosion limit of 40000ppm. It is therefore of safety for battery utilization, when the set-up pressure of safety valve and ventilation system were reasonable enough. Zhao et al. [22] have studied the hydrogen and oxygen evolution process of VRLA battery, and they found that the hydrogen evolution rate was increased by around $20 \%$ with per temperature in the range of $15-45^{\circ} \mathrm{C}$. Besides reaching the value of hydrogen explosion limit, a spark or heat source caused by short-circuit and thermal runaway could start battery fire as well.

\section{Fire prevention program of VRLA battery}

This section analyzes the relationship between VRLA battery failure and fire occurrence, and divides the fire evolution process into three stages: (1) Early battery failure; (2) Occurrence of thermal runaway, short circuit or hydrogen explosion; (3) Reaching the ignition temperature and then starting to burn. Based on the abovementioned theory, only one of these stages lost can prevent the fire occurrence. Details of the fire prevention scheme were descripted as follow.

\subsection{Improve battery performance}

Battery thermal safety has been studied by previous researches, while there are some significant factors, such as battery capacity, service life and economy still need comprehensive consideration in industrial application. The results by Sun et al. $[23,24]$ have found that added $0.05 \%$ to $1 \%$ oil, lignin, stearic acid or its stearate to negative electrode plate can significantly increase thermal runaway temperature of battery. Zhao et al. [25] studied that substitution the AGM separator or PVC separator can not only reduce the reaction rate of closed oxygen cycle, but also prevent the formation of lead dendrites and short circuit. In addition, Shao et al. [26] have studied the flame retardant properties of VRLA battery, and they found that the ABS resin was a flammable material with oxygen index from 18.3-20 $(<21)$. They have also identified that the addition of fumarate, polyvinyl chloride (PVC), halogen compounds and phosphorus-nitrogen flame retardants could improve the thermal resistant of battery significantly.

\subsection{Failed battery repair}

The researches by Yao et al., [27] Xie et al., [28] Tian et al., [29] showed that the failure modes such as "Sulfation of negative electrode plate", "Corrosion of positive electrode plate", "Battery "water loss" can be repaired in time by the following schemes. The "sulfation" failure usually accompanied by decreased battery capacity, which can be repaired with 2 4 times charge-discharge cycle, and complemented with "hydrotherapy desulfurization method" and "chemical desulfurization method". The principle is to convert the $\mathrm{PbSO}_{4}$ crystals in to active $\mathrm{Pb}$ and $\mathrm{PbO}_{2}$, by adjusting the times for charge-discharge cycle and electrolyte concentration. To avoid the corrosion of positive electrode plate, Xie et al. [27] have proposed to add some metal additives and conductive inorganic compounds to the $\mathrm{Pb}$ alloy, which in turn increasing the corrosion resistance and active material content. As for the "water loss" of battery, attributing to incomplete internal gas circulation, low setting pressure of safety valve, high floating voltage, which can be repaired by supplying water timely [28].

\subsection{Notes for users}

According to the $<<$ State Grid DC Power System Operation Specification $>>$ in china, the proper working temperature for VRLA battery ranges from $20-25^{\circ} \mathrm{C}$. The maximum temperature and the minimum distance between per battery should be $35^{\circ} \mathrm{C}$ and $10 \mathrm{~mm}$, respectively. Previous researchers [10] found that the floating voltage value should be corrected with the ambient temperature and the floating voltage of battery should be reduced by $3 \mathrm{mv}$ with per temperature increment. Moreover, a high-performance temperature detection system combined with automatic controlling system can be used to avoid battery thermal runaway. Users should also check the tightness of safety valve and set a proper 
safety pressure, since a large amount of hydrogen and oxygen will be kept inside the battery with high safety pressure, possibly causing explosion. However, the low set-up pressure will result in gas evolution and acid leakage, which in turn leading to battery "water loss".

\section{Conclusions}

The paper discusses several common failure modes of VRLA battery, analyses the correlation between failures and fire occurrence, and then proposes a fire prevention program accordingly. The results identify that battery failures such as "Sulfation of negative electrode plate", "Corrosion of the positive electrode plate", "water loss of battery" and "Acid leak of battery" increase the battery resistance as well as the Joule heat. Either the corrosion of positive electrode plate or "water loss" of battery decreases the capacity of VRLA battery, resulting in the battery thermal runaway easier. Furthermore, "Sulfation of negative electrode" and "Corrosion of the positive electrode" bend the plate, increasing the risk of battery short circuit. The thermal runaway of battery may flow out the acid electrolyte, corrode the circuit, and bring in short circuit. Although the research shows that the hydrogen concentration is difficult to reach the explosion limit under normal operation conditions, while the spark caused by short circuit or thermal runaway may ignite the mixture gas of hydrogen and oxygen around the battery. At last, a fire prevention scheme is proposed according to the "Three Combustion Factors", and thereby proposing program consist of "Improve battery performance", "Failed battery repair", "Notes for users" to decrease the possibility of battery fire.

\section{Acknowledgments}

Authors wishing to acknowledge the financial supports from the Science and Technology Project Funding of State Grid Corporation (Contract Number: 520530190006).

\section{References}

1. Jinquan W, Bing Z, Yongsong Y. (1995) Test and analysis of explosion of lead-acid battery for starting [J]. Fire Science \& Technology, (04):44-45(in Chinese).

2. Guang-fu W. (2012) Fire investigation and identification of a battery compartment fire[J]. Fire Science \& Technology, 31(11):1249-1251 (in Chinese).

3. Mariani A, Thanapalan K, Stevenson P, et al. (2013) Techniques for estimating the VRLA batteries ageing, degradation and failure modes[C]// International Conference on Automation \& Computing. IEEE.

4. Bouabidi, Ayadi, Kossentini, et al. (2016) Cycling Performances and Failure Modes for AGM and Standard Flooded Lead Acid Batteries under Partial State of Charge Mode[J]. Journal of energy engineering,142(3):04015027-1-04015027-7.
5. Valenciano J, A. Sánchez, Trinidad F, et al. (2006) Graphite and fiberglass additives for improving highrate partial-state-of-charge cycle life of valveregulated lead-acid batteries[J]. Journal of Power Sources, 158(2):851-863.

6. Qiu-hong W, Hua-qing WANG. (2005) On the failure modes of VRLA batteries and their influence factors[J]. Chinese Labat Man, (03):108-111 (in Chinese).

7. Guo-Rong C, Zheng-Kuang H. (2013) Research on fault prediction method of valve-regulated lead-acid battery based on internal resistance $[\mathrm{J}]$. Mechanical and Electrical Information, (18):37-38 (in Chinese).

8. Ball R J, Kurian R, Evans R, et al. (2002) Failure mechanisms in valve regulated lead/acid batteries for cyclic applications[J]. Journal of Power Sources, 109(1):189-202.

9. Wei C. (2012) Operation, maintenance and troubleshooting of valve-regulated sealed lead-acid battery for power [J]. Electric Engineering, (11):5-7 (in Chinese).

10. Qi Y, Yang X. (2009) Maintainance on Substation Valve Control Type Lead Acid Accumulator[J]. Hub Electric Power, 33(02):14-15+46 (in Chinese).

11. Hu J, Guo Y, Zhou X. (2006) Thermal runaway of valve-regulated lead-acid batteries $[\mathrm{J}]$. Journal of Applied Electrochemistry, 36(10):1083-1089 (in Chinese).

12. Pei-Chun W, Ke-Wen Z. (2002) The development trend of lead-acid storage batteries in the first ten years of the new century $[\mathrm{J}]$. Marine Electric \& Electronic Engineering, (02):46-55+64 (in Chinese).

13. Zhen-Ze H, Hong-Yu C, Jian Z. (2004) Failure and Maintenance for Valve-Regulated Lead-Acid Batteries[J]. The World of Power Supply, (12):46-49 (in Chinese).

14. Berndt D. (2001) Valve-regulated lead-acid batteries[J]. Journal of power sources, 95(1/2):p.2-12.

15. Meng T. (2014) Analysis and research on causes of fire lead-acid battery system[D]. Shenyang University of Technology, (in Chinese).

16. Zhi-Xue C, Ya-Hui L, Ke-Chan L. (2010) Discussion on short circuit of PE separators for lead acid batteries [J]. Chinese Labat Man,47(04):154-157 (in Chinese).

17. Bo-Yun L, Bo Z, Xiang-Lie Y I, et al. (2017) Gas evolving characteristics of submarine battery in the float-charging process without temperature compensation[J]. Fire Science and Technology, 36(01):23-26 (in Chinese).

18. JunY, Cheng H, Hao W. (2018) Research progress of failure model and mechanism analysis of lead-acid battery J]. Chinese Journal of Power Sources ,42(03):459-462 (in Chinese).

19. Lam L T, Haigh N P, Phyland C G, et al. (2004) Failure mode of valve-regulated lead-acid batteries under high-rate partial-state-of-charge operation[J]. Journal of Power Sources, 133(1):126-134.

20. Xuemei L, Weishan L I. (2003) The influence of 
bismuth on hydrogen and oxygen evolution behavior in lead-acid battery[J]. Battery Bimonthly, (01):55-57 (in Chinese).

21. Yantong W, Kaili X, Hui Z. (2016) Study on the distribution rule of hydrogen concentration in battery charging area [J]. Safety \& Security,37(10):32-35+39 (in Chinese).

22. Jian Z, Fan-Lin B O. (2002) Study on premature failure of VRLA batteries[J]. Battery Industry, (Z1):156-159 (in Chinese).

23. Cheng S. (2003) A review of thermal runaway in VRLA battery and countermeasures[J]. Chinese Labat Man, (03):134-136 (in Chinese).

24. Xiaoxiang S, Jianxi Z. (2017) Discussion on the thermal runaway in gel lead-acid battery[J]. Energy Storage ence and Technology,6(01):122-126 (in Chinese).
25. Jian-Xi Z, Bin Y, Xiao-Xiang S. (2015) Discussion about AGM/GEL hybrid battery[J]. Chinese Labat Man, 52(06):295-298 (in Chinese).

26. Shuangxi S. Study on the performance of flame retardant container of VRLA battery [J]. Chinese Labat Man,2013,50(05):213-216+221 (in Chinese).

27. Si-Chuan R. (2008) Processing and analysis of sulfation malfunction in lead-acid battery[J]. Telecommunications for Electric Power System,25(S1):120-121+131 (in Chinese).

28. XIE Xiao-yun, LIU Yue-jing, XIANG Bai-lin. (2010) Research progress in lead-acid battery grid material in China[J]. Battery Bimonthly, 40(3):173-176 (in Chinese).

29. Tengfei T, Yuan Y, Yangjun H. (2017) Discussion on Water Loss of UPS Valve-Controlled Sealed Lead Acid Battery [J]. Practical Electronics, (Z1):49-50 (in Chinese). 